\title{
Bovine glycomacropeptide promotes the growth of Bifidobacterium longum ssp. infantis and modulates its gene expression
}

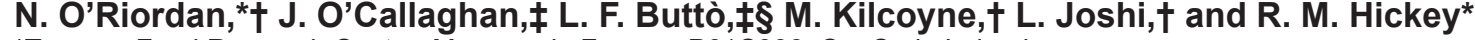 \\ *Teagasc Food Research Centre, Moorepark, Fermoy, P61C996, Co. Cork, Ireland \\ †Advanced Glycoscience Research Cluster, National Centre for Biomedical Engineering Science, National University of Ireland Galway, \\ H91TK33 Galway, Ireland \\ $\ddagger$ Department of Microbiology, and \\ $\S$ Alimentary Pharmabiotic Centre, University College Cork, T12K8AF Cork, Ireland
}

\section{ABSTRACT}

Bovine milk glycomacropeptide (GMP) is derived from $\kappa$-casein, with exclusively o-linked glycosylation. Glycomacropeptide promoted the growth of Bifidobacterium longum ssp. infantis in a concentration-dependent manner, and this activity was lost following periodate treatment of the GMP (GMP-P), which disables biological recognition of the conjugated oligosaccharides. Transcriptional analysis of B. longum ssp. infantis following exposure to GMP revealed a substantial response to GMP relative to bacteria treated with GMP-P, with a greater number of differentially expressed transcripts and larger fold changes versus the control. Therefore, stimulation of B. longum ssp. infantis growth by GMP is intrinsically linked to the peptide's $O$-linked glycosylation. The pool of differentially expressed transcripts included 2 glycoside hydrolase (family 25) genes, which were substantially upregulated following exposure to GMP, but not GMP-P. These GH25 genes were present in duplicated genomic islands that also contained genes encoding fibronectin type III binding domain proteins and numerous phage-related proteins, all of which were also upregulated. Homologs of this genomic arrangement were present in other Bifidobacterium species, which suggest it may be a conserved domain for the utilization of glycosylated peptides. This study provides insights into the molecular basis for the prebiotic effect of bovine milk GMP on B. longum ssp. infantis.

Key words: glycomacropeptide, Bifidobacterium, bovine milk, transcription

\section{INTRODUCTION}

A bifidobacteria-rich microbiome has numerous biological benefits to the host, including maintenance of

Received January 26, 2018.

Accepted April 6, 2018.

${ }^{1}$ Corresponding author: rita.hickey@teagasc.ie a healthy gastrointestinal tract, inhibition of microbial infection, and alleviating symptoms associated with digestive illness (Leahy et al., 2005; Picard et al., 2005). Therefore, interest is growing in enhancing the bifidobacterial population of the gastrointestinal tract. Human milk oligosaccharides (HMO) represent the main impetus for bacterial colonization of the distal large intestine of the breast-fed infant (Scholtens et al., 2012). The high concentrations of HMO and oligosaccharides either $\mathrm{N}$ - or $\mathrm{O}$-linked to proteins processed after intestinal digestion are thought to be the main contributors to the predominance of Bifidobacterium species in the infant gut (Garrido et al., 2013a). Indeed, the genome of one particular strain, Bifidobacterium longum ssp. infantis ATCC15697, a common member of the gastrointestinal microbiota of breast-fed infants, has revealed particular adaptations for the metabolism of HMO (LoCascio et al., 2007, 2010; Sela et al., 2008, 2011, 2012; Garrido et al., 2011, 2012b; Kim et al., 2013), milk glycoconjugates (Garrido et al., 2013a), and even commercial oligosaccharides such as galactooligosaccharides (Garrido et al., 2013b), and fructooligosaccharides (Perrin et al., 2001). In particular, the strain consumes these various carbohydrates using a variety of glycosyl hydrolases and $\mathrm{ABC}$ transporters (Sela et al., 2008; Garrido et al., 2013a).

However, the large quantities of HMO required for use as functional ingredients are unavailable, whereas commercial oligosaccharides such as galacto-oligosaccharides and fructo-oligosaccharides and individual HMO cannot match the complexity of the HMO pool present in breastmilk in terms of biological benefits. Bovine milk is potentially an alternative source of complex oligosaccharides with associated biological activity. However, the concentration and number of oligosaccharides is much lower than that of human milk, and currently the large quantities required are commercially unavailable. Certain glycoconjugates present in bovine milk have been purified at a large scale, as previously reviewed (O'Riordan et al., 2014), and may offer an 
alternative source of functional oligosaccharides. Karav et al. (2016) recently demonstrated the ability of bifidobacteria to use $N$-linked glycan chains cleaved from bovine milk glycoproteins as growth promoting factors and different bifidobacterial strains selectively consumed varying glycan structures.

Of the glycosylated proteins present in bovine milk, glycomacropeptide (GMP), a 64-AA peptide derived from $\kappa$-CN (Delfour et al., 1965), offers great potential as a functional food ingredient. Importantly, GMP can be produced in the quantities required for use as a food ingredient (Tullio et al., 2007) and it is commercially available as an ingredient for application in functional and medical foods, beverages, cosmetics, and supplements (O'Riordan et al., 2014; Ney et al., 2016). Glycomacropeptide has been formulated into medical foods for the treatment of phenylketonuria (Ney et al., 2016; Ney and Etzel, 2017) and has been shown to improve the growth of lactobacilli and bifidobacteria and enhance microbial diversity, both in vitro (Janer et al., 2004; Robitaille, 2013; Ntemiri et al., 2017) and in vivo (Chen et al., 2012; Sawin et al., 2015). Several other biological benefits have also been associated with GMP, including anti-infective (Nakajima et al., 2005; Gustavo Hermes et al., 2013) and antitoxigenic (Kawasaki et al., 1992; Isoda et al., 1989) activities. The carbohydrate content of bovine GMP is known to play a role in many of its associated biological benefits (O'Riordan et al., 2014).

However, reports are conflicting on the importance of the carbohydrate content of GMP in relation to the growth promotion of bifidobacteria, with recent studies suggesting that only the peptide backbone of CMP was essential for prebiotic activity (Robitaille, 2013). In the present work, the growth- and metabolic-related transcriptomic changes of an archetypical infant B. longum ssp. infantis strain following transient exposure to GMP is reported. The oligosaccharides on GMP were oxidized using sodium metaperiodate to investigate the role of GMP glycosylation. The observed phenotypic changes were subsequently correlated with transcriptional changes as determined by microarray analysis.

\section{MATERIALS AND METHODS}

\section{Materials}

Glycomacropeptide, with a maximum assayed lactose content of $1 \%$, was provided by Agropur Ingredients, (Eden Prairie, MN). Vivaspin 6 centrifugal filters with a 3-kDa molecular weight cutoff were purchased from Sartorius Stedim Biotech GmbH (Göttingen, Germany). Bifidobacterium longum ssp. infantis ATCC 15697 was purchased from DSMZ (Braunschweig, Germany). de Man, Rogosa, Sharpe (MRS) broth was purchased from Oxoid Ltd. (Basingstoke, UK). The Anaerocult A system was purchased from Merck (Darmstadt, Germany). All other reagents were from Sigma-Aldrich Co. (Dublin, Ireland), unless otherwise stated, and were of the highest grade available.

\section{Periodate Treatment of GMP}

Sodium metaperiodate $\left(\mathrm{NaIO}_{4}\right)$ treatment of GMP (GMP-P) was performed as previously described (Alemka et al., 2010). Briefly, GMP (2 mg/mL) was incubated with $0.011 \mathrm{mM} \mathrm{NaIO}_{4}$ (Alemka et al., 2010) at $4^{\circ} \mathrm{C}$ for $30 \mathrm{~min}$. Excess $\mathrm{NaIO}_{4}$ was removed by centrifugal filtration using a $3-\mathrm{kDa}$ molecular weight cutoff with 3 PBS, pH 7.4 washes and the retentate containing GMP-P was lyophilized.

\section{Bacterial Culture and Growth Experiments}

Bifidobacterium longum ssp. infantis was routinely cultured in MRS supplemented with $0.05 \%$ (wt/vol) Lcysteine (mMRS) at $37^{\circ} \mathrm{C}$ under anaerobic conditions generated using the Anaerocult A system (Kavanaugh et al., 2013). Bacterial culture stocks were maintained in mMRS containing $50 \% \mathrm{vol} / \mathrm{vol}$ glycerol at $-80^{\circ} \mathrm{C}$ and propagated twice in mMRS medium before use. Bacterial growth assays were performed in mMRS supplemented with increasing concentrations of GMP $(0.5$, $1,2,4$, and $8 \mathrm{mg} / \mathrm{mL}$ ) and filter sterilized through a $0.22-\mu \mathrm{m}$ membrane. The medium was inoculated with a $1 \% \mathrm{vol} / \mathrm{vol}$ inoculation of an overnight culture with an optical density at $600 \mathrm{~nm}\left(\mathbf{O D}_{600 \mathrm{~nm}}\right)$ of approximately 1.0 (corresponding to approximately $2 \times 10^{8} \mathrm{cfu} / \mathrm{mL}$ ). Cultures entered midexponential phase of growth after $16 \mathrm{~h}$, and $\mathrm{OD}_{600 \mathrm{~mm}}$ values at this time point were taken for comparison between each treatment. Growth experiments were performed in triplicate and the data presented is the average of 3 independent replicate assays. The $\mathrm{OD}_{600 \mathrm{~nm}}$ was measured on a PharmaSpec UV1700 UV-visible spectrophotometer (Shimadzu, Kyoto, Japan).

\section{RNA Isolation}

Bifidobacterium longum ssp. infantis was cultured to midexponential phase in mMRS as a control and mMRS supplemented with GMP or GMP-P (2 mg/ $\mathrm{mL}$ ) and bacterial pellets were harvested by centrifugation at $4,500 \times g$ for $8 \mathrm{~min}$ at $4^{\circ} \mathrm{C}$. The supernatant was removed and the bacterial pellets were resuspended in RNAprotect bacteria reagent (Qiagen, Hilden, Germany) and incubated at room temperature for $10 \mathrm{~min}$. Bacteria were again pelleted at $5,000 \times g$ for $10 \mathrm{~min}$ 
and the supernatant was discarded. Bacterial RNA was isolated using the RNA isolate mini kit (Bioline, London, UK) with modifications, as previously described (Kavanaugh et al., 2013). The RNA quantity and quality was determined using a NanoDrop 1000 (Thermo Fisher, Wilmington, DE), and products with 260/280 $\mathrm{nm}$ readings $>1.9$ and $230 / 260 \mathrm{~nm}$ readings $\geq 1.00$ were selected for further analysis. Sample quality was further assessed by agarose gel electrophoresis. The RNA samples were subsequently shipped to IMGM laboratories (Martinsried, Germany) for labeling and DNA microarray analysis. To ensure the absence of RNA degradation during transit, RNA was again assessed using an Agilent Bioanalyser (RNA600 Chip; Agilent Technologies, Co. Cork, Ireland) and only samples with a RNA integrity number (RIN) of 10 were approved for microarray analysis.

\section{Microarray Analysis}

The RNA was spiked with synthetic polyadenylated transcripts (Agilent spike-in controls) and labeled by a protocol for reverse transcription followed by in vitro transcription. In brief, $500 \mathrm{ng}$ of RNA was used for each reaction, which was reversed transcribed into cDNA using random priming (Full SpectrumTM MultiStart Primers for T7 IVT, System Biosciences, Palo Alto, CA). This was then converted into labeled cRNA by in vitro transcription (Quick-Amp Labeling Kit OneColor, Agilent Technologies) incorporating cyanine 3 (Cy3)-conjugated CTP. Manufacturer's instructions (https://www.agilent.com/cs/library/usermanuals/ Public/G4140-90040_GeneExpression_OneColor_6.9 .pdf) were followed for both the reverse transcription and labeling reactions.

A NanoDrop analyzer and an Agilent 2100 Bioanalyser with a 6000 Nano LabChip Kit (Agilent Technologies) were employed to determine the labeling efficacy and samples with cRNA yields $>825 \mathrm{ng}$ and a specific activity of $9.0 \mathrm{pmol}$ of $\mathrm{Cy} 3 / \mu \mathrm{g}$ of cRNA were selected for array analysis. Following cRNA clean-up and quantification (Thermo Fisher), $600 \mathrm{ng}$ of each Cy3-labeled cRNA sample was fragmented and prepared for OneColor based hybridization (Gene Expression Hybridization Kit, Agilent Technologies). Hybridization was performed at $65^{\circ} \mathrm{C}$ for $17 \mathrm{~h}$ on custom Bifidobacterium GE Microarrays $(8 \times 15 \mathrm{~K}$ format) as per Kavanaugh et al. (2013), which were then washed with increasing stringency using Gene Expression Wash Buffers (Agilent Technologies) followed by drying with acetonitrile. Fluorescent signal intensities were detected with Scan Control A.8.4.1 Software (Agilent Technologies) on the Agilent DNA Microarray Scanner and extracted from the images using Feature Extraction 10.7.3.1 Software (Agilent Technologies) and the design file 033172_D_F_20110831.xml.

\section{Quantitative Real-Time PCR Analysis}

Complementary DNA was synthesized from $1 \mu \mathrm{g}$ of RNA (isolated as described above) as per Kavanaugh et al. (2013). Template and random primers were incubated at $65^{\circ} \mathrm{C}$ for $10 \mathrm{~min}$, followed by addition of the remaining components. The reaction mixture was then incubated at $55^{\circ} \mathrm{C}$ for $30 \mathrm{~min}$ and inactivated by heating to $85^{\circ} \mathrm{C}$ for $5 \mathrm{~min}$. The PCR primers and probes were designed using the Universal Probe Library Assay Design Centre (Roche, Basel, Switzerland). Primer sequences and probe combinations are listed in Supplemental Table S1 (https://doi.org/10.3168/jds .2018-14499). The 16S rRNA (Blon_R0085) was used as an endogenous control to correct for variability in the starting total RNA and provide a stable expression marker against which relative levels of expression were determined. Amplification reactions were performed in duplicate as per Kavanaugh et al. (2013). The $2^{-\Delta \Delta \mathrm{Ct}}$ method (Livak and Schmittgen, 2001) was employed to calculate relative changes in gene expression.

\section{Computer Analysis}

The DNA sequence analyses and homology searches were carried out using the NIH BLAST program (Altschul et al., 1990).

\section{Statistical Analysis}

The microarray data were processed using the Limma package of the $\mathrm{R}$ statistics suite (http://www .R-project.org). Background correction was done using the normexp method with offset $=16$. Interarray normalization was not done. The microarray data were clustered using the heatmap. 2 package in $\mathrm{R}$ to identify any experimental samples as outliers. The fold change values presented are actual fold change values for the test samples relative to the controls. Genes with a fold change of $>1.5$ where $P \leq 0.05$ were considered to be differentially transcribed. Transcriptional changes were displayed graphically through the use of Volcano plots $\left(-\log _{10} P\right.$-value vs. $\log _{2}$ fold-change) and whole genome plots, where the fold change for each locus tag was plotted as a bar chart.

The statistical treatment of the quantitative realtime PCR (RT-qPCR) data was by Student's $t$-test and one-way ANOVA for pairwise or multiple compari- 
sons, respectively, to determine statistically significant results, where $P<0.05$ was considered significant.

\section{RESULTS AND DISCUSSION}

\section{Effect of GMP on B. longum ssp. infantis Growth}

To investigate if GMP had a prebiotic effect on the archetypal infant associated bifidobacterial strain, $B$. longum ssp. infantis was cultured in mMRS supplemented with $2 \mathrm{mg} / \mathrm{mL}$ of GMP, as this concentration has been proven to positively influence the growth of probiotic strains Lactobacillus rhamnosus RW-9595-M and Bifidobacterium thermophilum RBL67 (Robitaille, 2013). Higher concentrations are also unlikely to occur in biological conditions in vivo, as levels of 1.2 to 1.5 $\mathrm{mg} / \mathrm{mL}$ are found in cheese whey, which is almost 10 times higher than free GMP in mature milk (Furlanetti and Prata, 2003; Neelima et al., 2013). A $20.6 \pm 3.6 \%$ increase in $\mathrm{OD}_{600 \mathrm{~nm}}$ was observed in the midexponential phase of B. longum ssp. infantis cultures supplemented with GMP, indicating a growth promoting effect (Figure 1A). Assays were repeated with increasing GMP concentrations and $\mathrm{OD}_{600 \mathrm{~nm}}$ was recorded after $16 \mathrm{~h}$ of incubation, at the midexponential phase. A concentration-dependent growth-promoting effect was observed (Figure 1B), with the most significant effect (an increase of $44 \pm 9.9 \%$ versus the control) observed using $8 \mathrm{mg} / \mathrm{mL}$ of GMP. A concentration of $2 \mathrm{mg} / \mathrm{mL}$ of GMP was chosen for the remainder of the study, as this concentration was used in previous studies (Robitaille, 2013) and correlates with the concentration of GMP present in cheese manufacturing whey $(1.2-1.5 \mathrm{mg} / \mathrm{mL}$; Neelima et al., 2013).

Addition of $2 \mathrm{mg} / \mathrm{mL}$ of oxidized GMP-P resulted in a substantially lower $(5.5 \%)$ increase in growth compared with that following addition of $2 \mathrm{mg} / \mathrm{mL}$ of GMP (20.6\%; Figure 1C). Periodate oxidation of glycoproteins converts vicinal diols of oligosaccharide residues to 2 aldehyde groups, making the carbohydrate structures biologically unrecognizable (Kilcoyne et al., 2011). Following periodate treatment of GMP, a decrease of approximately $75 \%$ in the growth promotion effect of the glycopeptide was observed. A slight growth increase was still evident in the GMP-P group, indicating that the peptide portion may also influence bacterial growth. Overall, this data suggests that GMP glycosylation has a very important role to play in the promotion of $B$. longum ssp. infantis growth.

Glycomacropeptide is exclusively $O$-glycosylated with both neutral and acidic oligosaccharides (Saito, 1980; Saito et al., 1981a,b, 1982; Kawasaki et al., 1993). Sialic acid is a prominent feature of these glycans, with
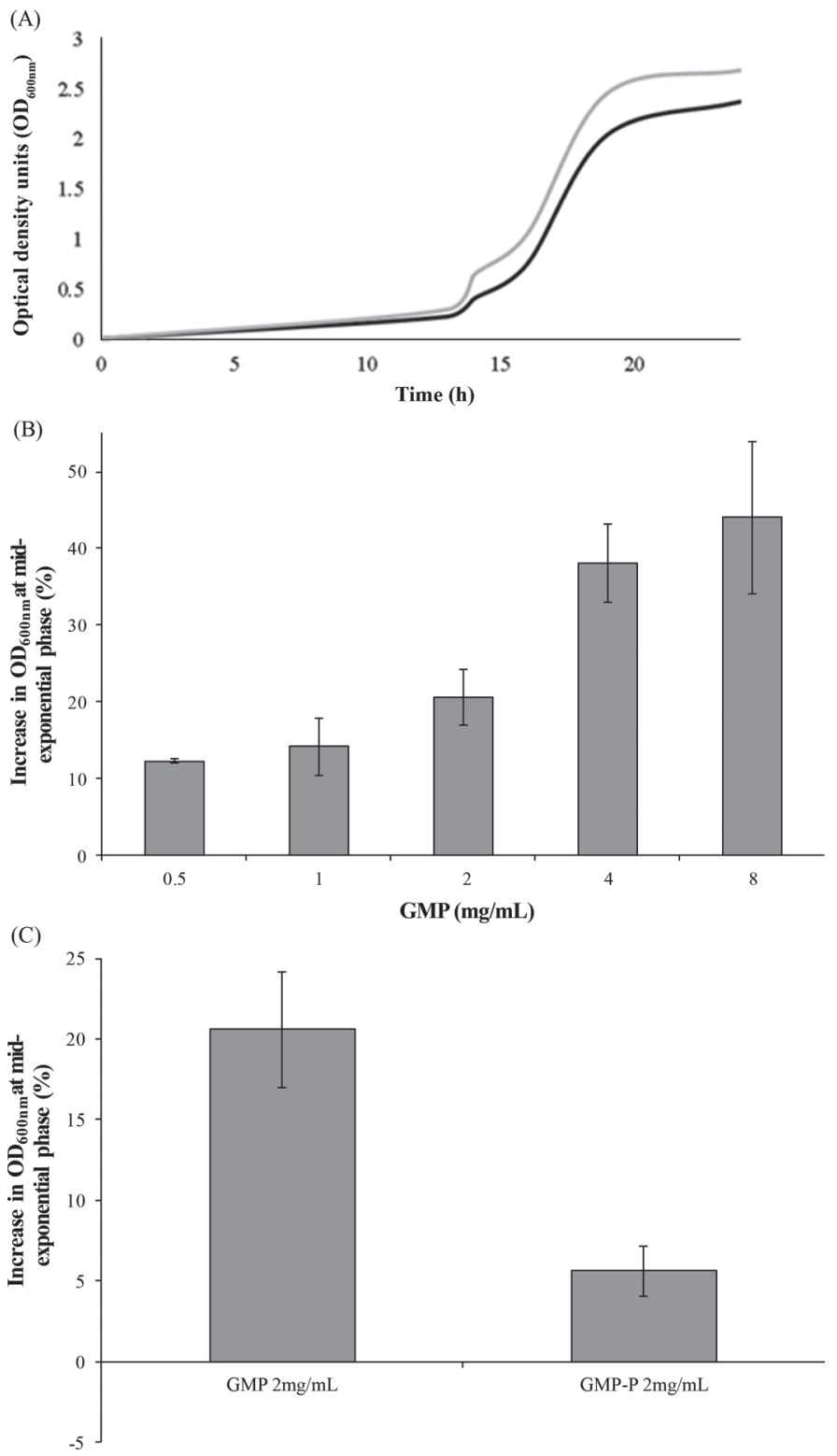

Figure 1. (A) Growth of Bifidobacterium longum ssp. infantis in de Man, Rogosa, and Sharpe broth supplemented with $0.05 \% \mathrm{wt} / \mathrm{vol}$ L-cysteine (mMRS) with (gray) and without (black) supplementation with $2 \mathrm{mg} / \mathrm{mL}$ of bovine glycomacropeptide (GMP) over $24 \mathrm{~h}$. (B) Percent increase in optical density at $600 \mathrm{~nm}\left(\mathrm{OD}_{600 \mathrm{~nm}}\right)$ of B. longum ssp. infantis cultured in the presence of increasing concentrations of GMP versus unsupplemented mMRS at mid-exponential phase. (C) Comparison of the percent increase in $\mathrm{OD}_{600 \mathrm{~mm}}$ of $B$. longum ssp. infantis cultured in the presence of $2 \mathrm{mg} / \mathrm{mL}$ of GMP or periodate treatment of the GMP (GMP-P) versus unsupplemented mMRS at midexponential phase. Error bars indicate \pm SD.

$82 \%$ of GMP oligosaccharides being either mono- or disialylated (Hua et al., 2012). This high sialic acid content may be the basis for the prebiotic activity described for GMP, as prebiotic activity has previously 
been linked to other sialic acid containing compounds (Idota et al., 1994).

\section{Gene Expression Analysis}

Bifidobacterium longum ssp. infantis was cultured to midexponential phase in MRS, MRS + GMP (2 $\mathrm{mg} / \mathrm{mL})$, and MRS + GMP-P (2 mg/mL) and bacteria were harvested for microarray analysis to profile gene expression in response to the presence of GMP or GMP-P in the growth medium versus the control (MRS only). The data were submitted to Gene Expression Omnibus (GEO) functional genomics data repository (Edgar et al., 2002), and assigned accession number GSE75969 with the array platform designated GPL21235. The overall number of genes differentially expressed following GMP supplementation is summarized in Figure 2A, and a complete list of differentially expressed transcripts (DET) is provided in Supplemental Table S2 (https://doi.org/10.3168/jds.2018-14499). The largest collection of DET were upregulated by GMP (264 genes), whereas considerably fewer genes were found to be downregulated (47 genes); 153 DET were upregulated by GMP-P and none were downregulated. The DET were categorized into groups based on their annotated function as genes involved in glycan metabolism, cellular transport, protein metabolism, cell division and growth, and hypothetical, or proteins of unknown function and other (Figure 2B). With the exception of the hypothetical protein category, genes involved in cell division were the largest population of DET, which is not surprising considering the observed growth promotion effect of GMP.

In total, 56 genes were shared between both GMP and GMP-P treatments (Figure 2A), with the majority of the annotated genes in this population involved in
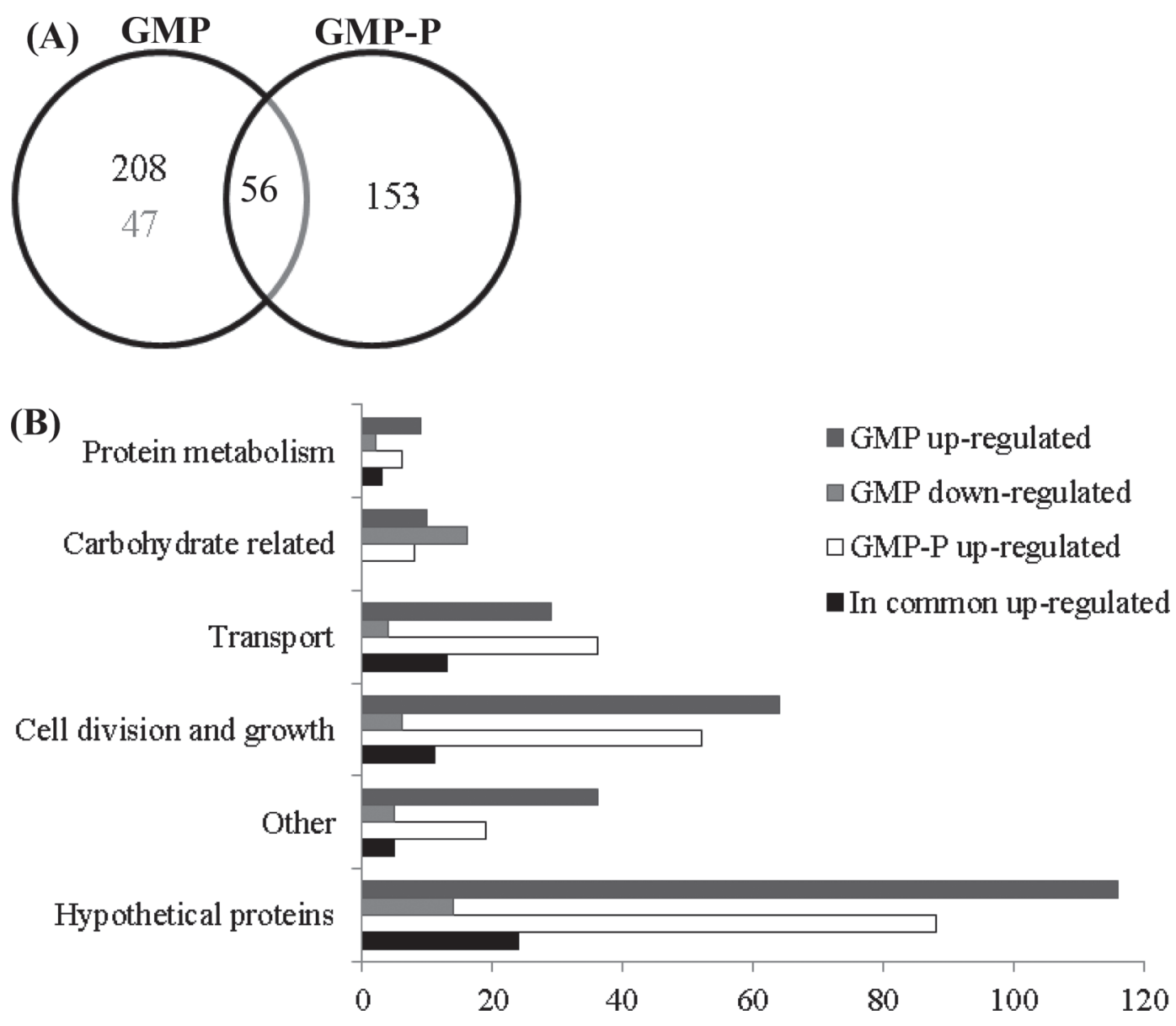

Figure 2. (A) Number of differentially expressed transcripts following exposure to glycomacropeptide (GMP) or oxidized periodate treatment of the GMP (GMP-P). The cutoff point for inclusion was fold change $\geq 1.5$ and $P \leq 0.05$. Black = upregulated versus control. Gray $=$ downregulated versus control. (B) Numbers of Bifidobacterium longum ssp. infantis genes, grouped according to functional category, which were differentially regulated following growth in the presence of GMP or GMP-P. Black bars indicated the number of genes that were common to both treatments. 
noncarbohydrate-related transport and cell growth, as outlined in Supplemental Table S2 (https://doi.org/10 .3168/jds.2018-14499). A cluster of phosphonate ABC transporter genes (Blon_0020-Blon_0025), as well as numerous other transport-related genes, were upregulated by both treatments and may give insight into the effect of GMP on nutrient uptake by $B$. infantis from the environment. When the genome of $B$. infantis was first published in 2008, 35\% of the ORFs in B. longum ssp. infantis were annotated as hypothetical proteins, which comprised 845 proteins of unknown function (Sela et al., 2008). The majority of these genes are still currently annotated as hypothetical, and this category represented the largest group of genes influenced by exposure to GMP and GMP-P, indicating the need for further functional genomic studies to elucidate the role of specific genes. Volcano plots (Figure 3) provide a graphic representation of the greater magnitude of the transcriptomic response to GMP treatment when compared with GMP-P. Although both treatments resulted in a substantial number of DET in comparison to the control, with 311 and 209 genes, respectively (Figure 2 ), the Volcano plots illustrated that the response to GMP was more extensive, with larger fold changes

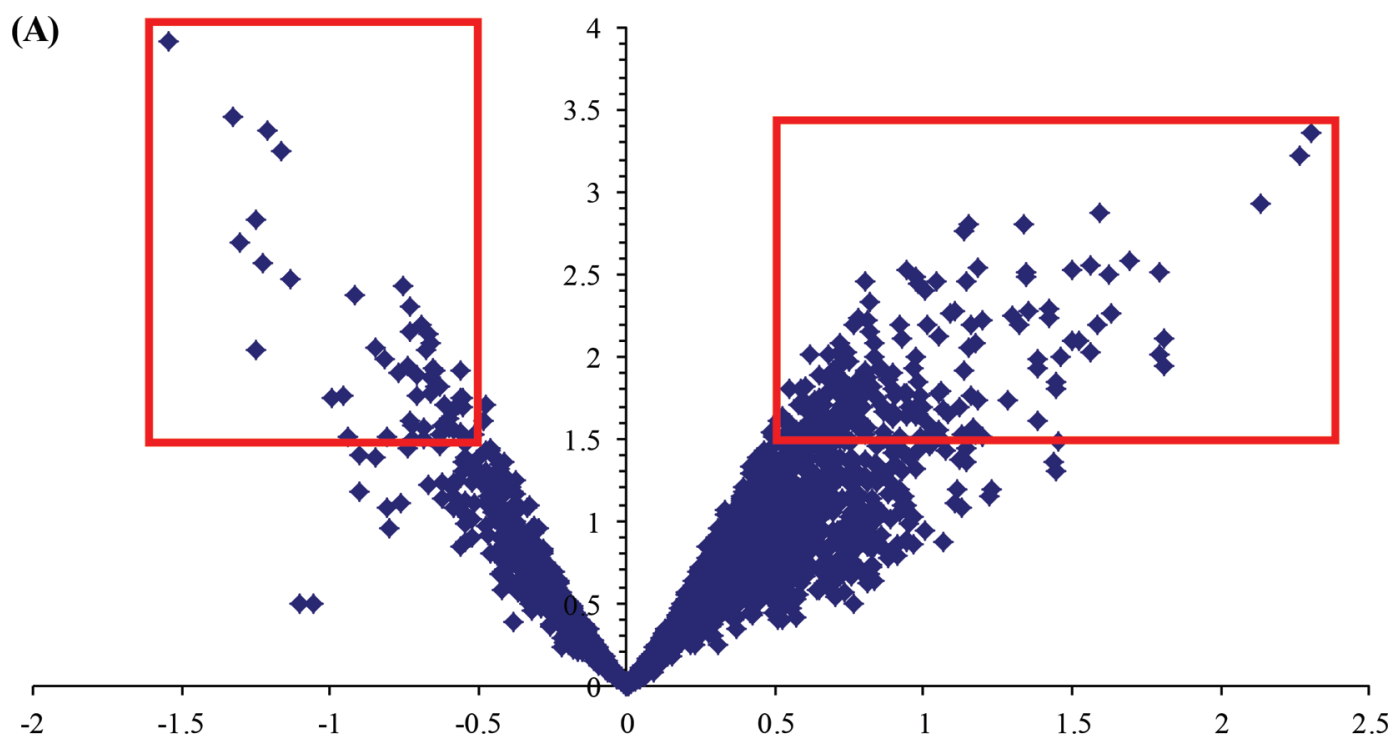

(B)

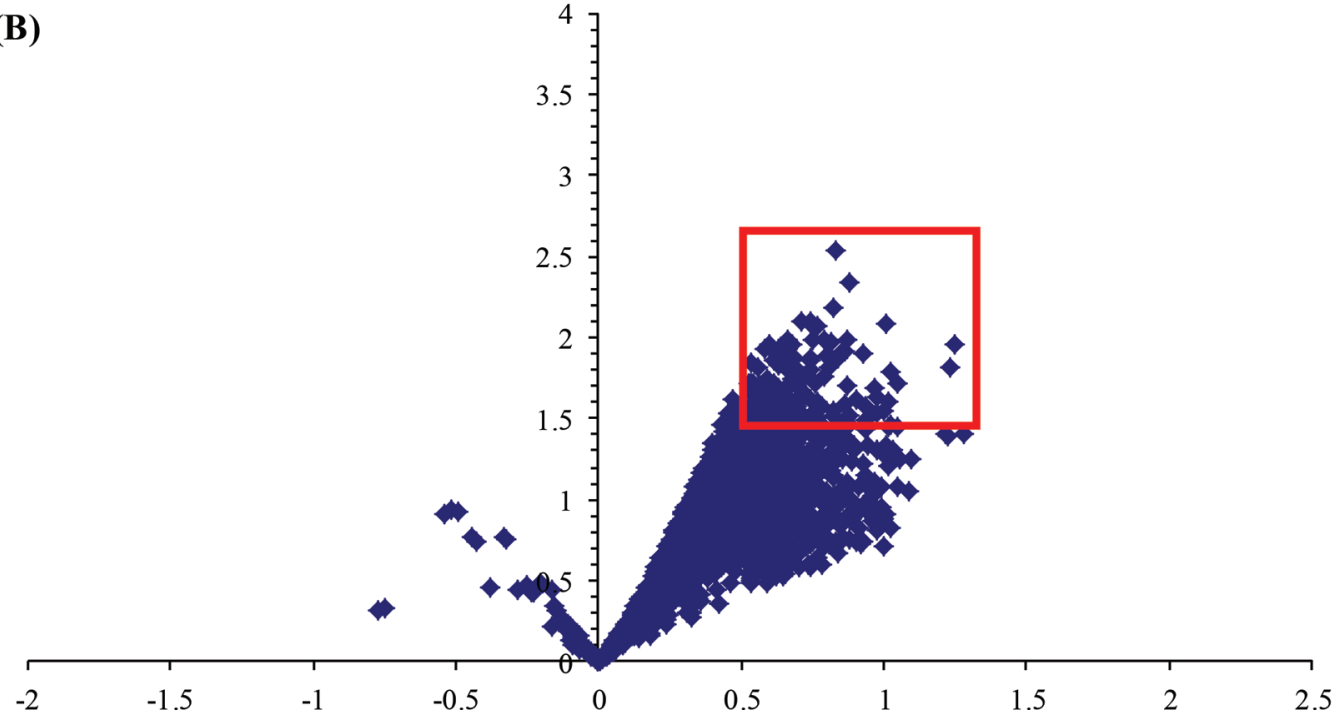

Figure 3. Volcano plots $\left[-\log _{10} P\right.$-value (y-axis) vs. $\log _{2}$ fold-change (x-axis)] demonstrating transcriptional changes following Bifidobacterium longum ssp. infantis exposure to (A) glycomacropeptide (GMP) and (B) periodate treatment of the GMP (GMP-P). Boxes highlight genes that were deemed as significantly changed following each treatment (fold change $\geq 1.5$ and $P \leq 0.05$ ). Color version available online. 
Table 1. Carbohydrate-related genes: differentially regulated genes associated with carbohydrate metabolism

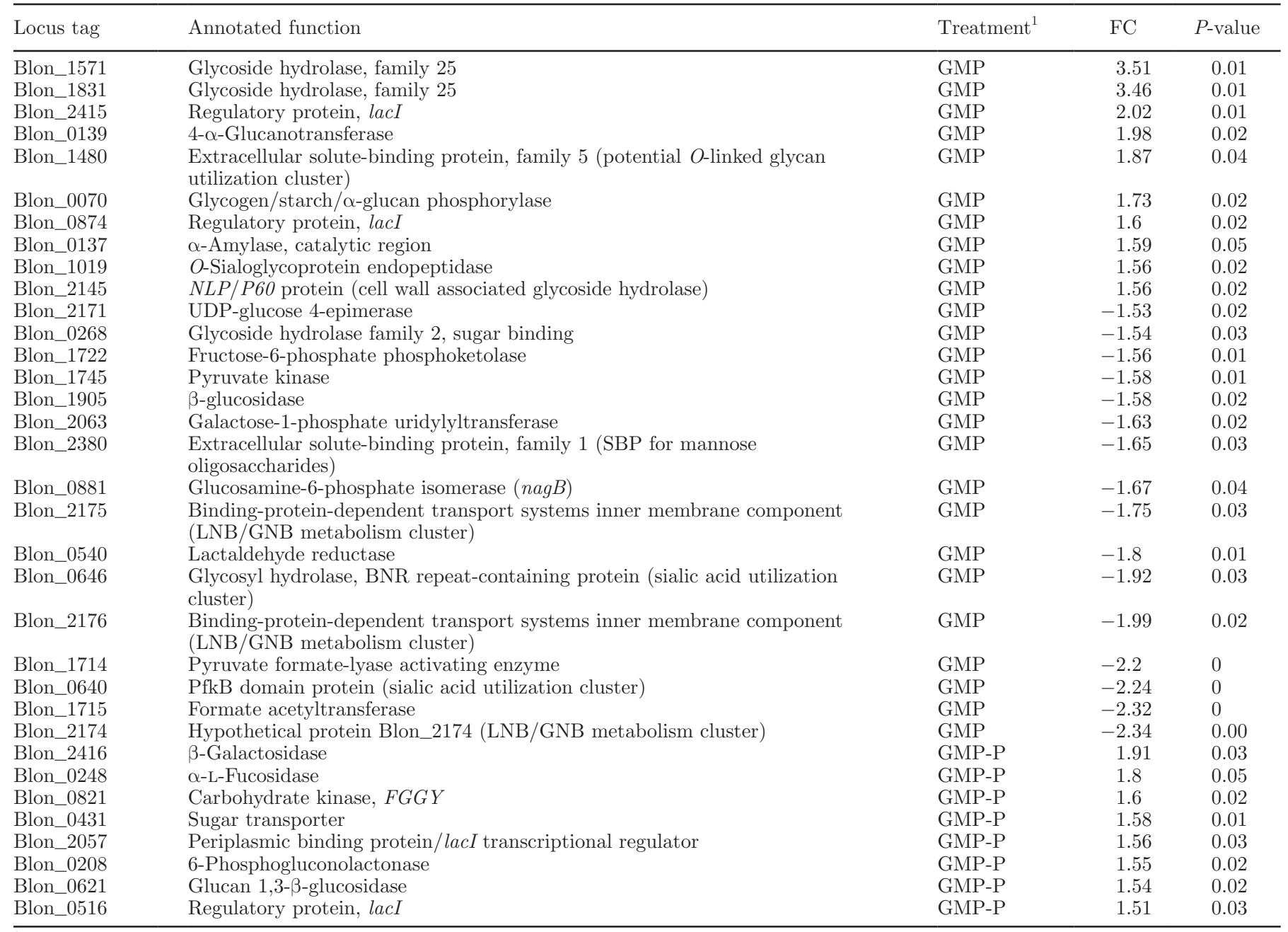

${ }^{1} \mathrm{GMP}=$ bovine milk glycomacropeptide; GMP-P = periodate treatment of the GMP; FC = fold change; UDP = uridine diphosphate glucose; $\mathrm{SBP}=$ solute binding protein; $\mathrm{LNB} / \mathrm{GNB}=$ lacto- $N$-biose/galacto- $N$-biose; $\mathrm{BNR}=$ bacterial neuraminidase repeat.

observed in differential expression overall. This finding suggests that the oxidation of the GMP oligosaccharides reduced the effect of GMP on B. longum ssp. infantis gene expression.

\section{Effect of GMP Treatment on Carbohydrate- Related Genes}

Glycomacropeptide treatment resulted in the upand downregulation of genes involved in carbohydrate transport and metabolism (Table 1). Although several carbohydrate metabolism genes were also differentially expressed in response to GMP-P, the genes were different to those upregulated in response to GMP-P and the fold change in expression was generally lower (Table 1). Several genes annotated as lacI regulatory genes were upregulated by both GMP and GMP-P. The lactose operon repressor (LacI) proteins can interact directly with DNA and inhibit expression of genes involved in lactose metabolism and the genes annotated as lacI genes in Table 1 may have a similar role in regulating sugar metabolism in B. longum ssp. infantis if functional. Although unique lacI regulatory genes were differentially expressed in response to both treatments, those upregulated in the GMP-P group were induced to a much lesser extent. This differential induction of regulatory genes suggested that $B$. longum ssp. infantis may modulate its carbohydrate metabolism pathways in response to the GMP oligosaccharides.

Of the carbohydrate-related genes upregulated solely in response to GMP-P, the most significant were a $\beta$-galactosidase (Blon_2416) and an $\alpha$-L-fucosidase (Blon_0248). The increased expression of a fucosidase gene was unexpected, as fucosylated GMP oligosaccha- 
rides have only been detected in GMP isolated from colostrum to date (Fournet et al., 1975) and mature milk was the source of the GMP used here. In addition, GMP-P was periodate treated, so intact fucose residues should not be present, regardless of whether they were present or not before periodate treatment. The expression of an $\alpha-\mathrm{L}-$ fucosidase in $B$. longum ssp. infantis during growth on inulin, a nonfucose-containing substrate, has previously been reported also (Kim et al., 2013). The expression of $\beta$-galactosidase (Blon_2416) was also increased in the GMP group (fold change $=1.65$ ), but was not statistically significant $(P=0.07)$. However, numerous genes in close proximity to Blon_2416 on the chromosome were also upregulated following growth in GMP (Blon_2415, Blon_2419-Blon_2423; Supplemental Table S2, https://doi.org/10.3168/jds.2018-14499), including a lacI regulatory gene.

Expression of 3 genes related to sucrose and starch metabolism were upregulated following exposure to GMP only; Blon_0070, Blon_0137 and Blon_0139. These genes may be involved in the metabolism of glucose present in the growth media. Their increased expression following exposure to GMP could result in an increased metabolism of glucose from the environment and contribute to the observed growth increase. Interestingly, Blon_0139 was also upregulated following exposure of $B$. longum ssp. infantis to a mixture of $3^{\prime}$ - and 6 '-sialyllactose (Kavanaugh et al., 2013). The presence of a possible $O$-linked oligosaccharide utilization gene cluster on the B. longum ssp. infantis genome was previously predicted (Garrido et al., 2013a) and comprised Blon_1479, an $\alpha-N$-acetylgalactosaminidase homolog, and Blon_1480, an ABC transporter for peptide importation. In the present study, Blon_1480 was highly upregulated in response to growth in the presence of GMP whereas Blon_1479 expression was unaffected. As GMP is characterized by highly sialylated $O$-linked oligosaccharides, it may be recognized by Blon_1480 for import. Furthermore, the gene Blon_1019 was also upregulated following growth in the presence of GMP and it has a predicted function in the metabolism of $O$-glycosylated peptides as an $O$-sialoglycoprotein endopeptidase.

Two candidate sialidase genes have previously been identified within the B. longum ssp. infantis genome, Blon_0646 and Blon_2348, which appear to be intracellular (Sela et al., 2008). The first gene is present in a gene cluster dedicated to sialic acid metabolism, which provides $\mathrm{N}$-acetyl glucosamine-6-P for further processing into fructose-6-P to enter central metabolism (Sela et al., 2011). Blon_0646 was downregulated in the GMP group in addition to related genes, Blon_0640, Blon_1722 (fructose-6-P phosphoketolase), Blon_1745 (pyruvate kinase), and Blon_0881 (nagB). For degradation of HMO, B. longum ssp. infantis deploys a utilization strategy involving translocation of intact oligosaccharides using $\mathrm{ABC}$ transporters. Once internalized, milk oligosaccharides are degraded by several intracellular glycosyl hydrolases that target linkages inherent to HMO. Released monosaccharides are fermented in the central fructose-6-phosphate phosphoketolase pathway (bifid shunt), resulting in the production of ATP and secretion of acetate and lactate as end-products (Garrido et al., 2015). Given that GMP is a more complex carbohydrate source, it may be that $B$. longum ssp. infantis diverts some of its energy into producing the solute binding proteins and glycosyl hydrolases required for degradation of GMP at the expense of the simpler disaccharide and monosaccharide metabolism during this phase of growth. When grown on complex carbohydrates, bifidobacteria may improve their fitness if able to use such sources, despite the lower growth rate when compared with simple carbohydrates.

A recent study by Karav et al. (2016) on $N$-linked glycoproteins from whey supports this hypothesis. Here, B. longum ssp. infantis ATCC 15697 did not grow on the deglycosylated milk protein fraction, clearly demonstrating that the glycan portion of the milk glycoproteins provided the key substrate for growth. The authors found that an endoglycosidase (EndoBI-1) is first required to carry out the initial deglycosylation and that EndoBI-1 expression is based on the glycan portion of the glycoprotein. Given that free milk oligosaccharides do not induce EndoBI-1 expression (Garrido et al., 2015), this suggests that B. longum ssp. infantis senses intact glycoprotein and induces EndoBI-1 in response. As GMP is $O$-glycosylated, upregulation of $O$-endoglycosidase(s) such as Blon_1019 would be expected. The fact that no change in regulation of the other sialidase, Blon_2348, or in genes such as Blon_2016 ( $\beta$-galactosidase) was observed in the presence of GMP may not be surprising, as these enzymes that have been linked with the degradation of free glycans (HMO; Garrido et al., 2012a).

Interestingly, expression of several other genes related to simple sugar and free oligosaccharide metabolism, such as members of the lacto- $N$-biose (LNB) and galacto- $N$-biose (GNB) metabolism cluster (Blon_2171-Blon_2177; LoCascio et al., 2010), were downregulated in the GMP-treated group. The downregulation of the same genes in the LNB/GNB cluster (Blon_2174-Blon-2176) was previously described following exposure of $B$. longum ssp. infantis to $3^{\prime}$ - and 6 '-sialyllactose (Kavanaugh et al., 2013). No significant transcriptional changes were observed on the $43-\mathrm{kbp}$ cluster at Blon_2331-Blon_2361, which are dedicated 
to the import and processing of HMO (Sela et al., 2008). Downstream of this genome region, a large number of genes were differentially expressed in the GMP-treated group, including upregulation of Blon_2370-Blon_2373, involved in bacterial growth, and downregulation of transport-related Blon_2379-Blon_2380. Again, a similar effect was previously observed following exposure to 3'- and 6'-sialyllactose (Kavanaugh et al., 2013).

Two genes encoding glycoside hydrolases from family 25 (GH25), Blon_1571 and Blon_1831, were upregulated in response to GMP. Family 25 has only 1 documented function to date, as a lysozyme involved in cell wall and peptidoglycan catabolism, and may thus have a role in facilitating cell division and in defense. When the whole-genome transcription plots were considered, large peaks in the GMP-exposed cells were observed in the vicinity of both significant GH25 (Supplemental Figure S1 https://doi.org/10.3168/jds.2018-14499). When these genetic loci were studied in more detail, clusters of substantial homology were identified. The cluster at Blon_1546 - Blon_1571 (cluster A) had a larger number of genes displaying increased expression, with the highest overall upregulation observed for hypothetical genes at this locus. Fibronectin type III domain proteins (Fn3) were also present in both clusters downstream of the GH25 (Blon_1563 and Blon_1825). Both Fn3 proteins were also upregulated in response to GMP, as were several other hypothetical genes in the clusters. The Fn3 domains have previously been described in several bacterial glycoside hydrolases (Little et al., 1994; da Silva et al., 2014). These domains may have a role in the degradation of insoluble substrates and may have been acquired by bacteria through horizontal gene transfer from animals (da Silva et al., 2014).

A BLAST search (https://blast.ncbi.nlm.nih.gov/ Blast.cgi) for similar Fn3 proteins identified 2 other bifidobacteria-containing gene arrangements similar to cluster A of B. longum ssp. infantis. Bifidobacterium breve DSM20213 had just 1 region of homology with a Fn3 protein at locus BIFBRE_01730, whereas Bifidobacterium adolescentis L2-32 contained 2 duplicates of the cluster with Fn3 proteins at loci BIFADO_01393 and BIFADO_01092. The GH25 hydrolases were only present in the clusters identified in B. longum ssp. infantis, and only the genes encoding for Fn3 protein and both flanking genes showed homology across all 5 domains (Figure 4). A BlastX (https://blast.ncbi.nlm.nih .gov/Blast.cgi?LINK_LOC=blasthome\&PAGE_TYPE $=$ BlastSearch\&PROGRAM=blastx) analysis revealed all Fn3 proteins shared $\geq 50 \%$ identity (Supplemental Table S3 https://doi.org/10.3168/jds.2018-14499). Clustal analysis revealed large areas of homology between all 5 Fn3 proteins at the N- and C-termini, including the fibronectin type III domain. These genome comparisons suggest that some bifidobacteria may

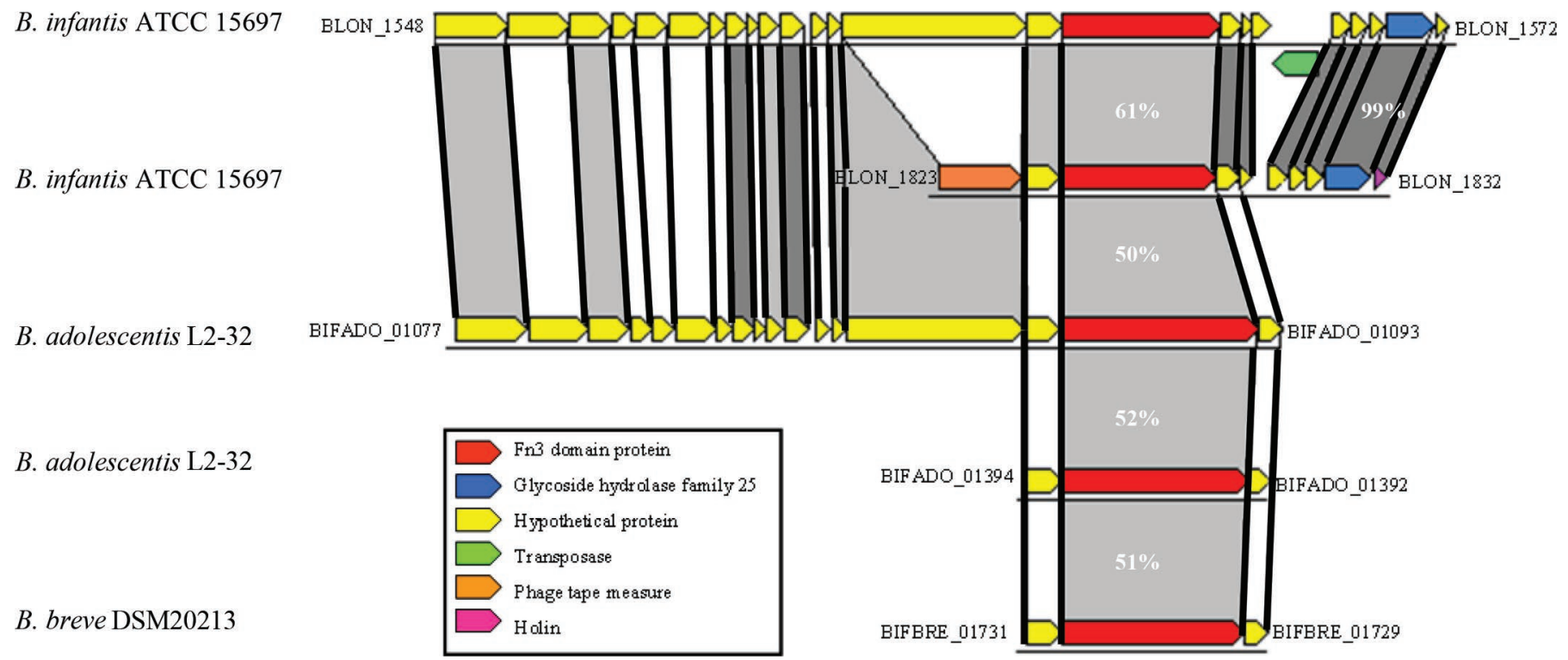

Figure 4. Comparison of clusters identified in Bifidobacterium containing fibronectin type III domain proteins. Each solid arrow indicates an open reading frame (ORF) and arrow length is proportional to the length of the predicted ORF. Locus tags are indicated at the beginning and end of areas of homology and arrow color indicates annotated function. The AA identity of each predicted protein is indicated as a percentage relative to its equivalent protein encoded by Bifidobacterium longum ssp. infantis ATCC 15697 in the cluster present at locus tags blon_1548blon_1572 (white shading $=25-50 \%$ identity, light gray shading $=50-70 \%$ identity, dark gray shading $=>70 \%$ identity). 
Table 2. Summary of fold changes following growth in the presence of GMP and GMP-P, ${ }^{1}$ as determined by microarray and quantitative realtime PCR (RT-qPCR) analysis

\begin{tabular}{|c|c|c|c|c|}
\hline \multirow[b]{2}{*}{ Locus tag and annotated function } & \multicolumn{2}{|c|}{ GMP } & \multicolumn{2}{|c|}{ GMP-P } \\
\hline & Array & RT-qPCR & Array & RT-qPCR \\
\hline Blon_0646 glycosyl hydrolase, BNR repeat-containing protein & -1.92 & -6.45 & - & -1.53 \\
\hline Blon_1555 hypothetical protein & 4.38 & 4.78 & - & 1.00 \\
\hline Blon_1562 hypothetical protein & 3.47 & 5.71 & - & 1.03 \\
\hline Blon_1563 fibronectin, type III domain protein & 2.25 & 6.51 & - & 1.00 \\
\hline Blon_1825 fibronectin, type III domain protein & 2.72 & 6.08 & - & -1.08 \\
\hline Blon_2421 binding-protein-dependent transport systems inner membrane component & 2.68 & 2.63 & - & -1.06 \\
\hline Blon_2423 bleomycin hydrolase & 3.02 & 3.05 & - & 1.41 \\
\hline
\end{tabular}

${ }^{1} \mathrm{GMP}=$ bovine milk glycomacropeptide; GMP-P = periodate treatment of the GMP.

${ }^{2}$ Indicates values that were determined insignificant due to their high $P$-value. A $P$-value $\leq 0.05$ is considered significant.

contain gene clusters that allow them to use complex glycopeptide substrates such as GMP.

\section{Gene Expression Validation by RT-qPCR}

Due to the high similarity between the genes in the both GH25 clusters, RT-qPCR was performed to verify both clusters were upregulated and eliminate the possibility of cross-hybridization on the array resulting in false positives. Here, the expression levels of the $16 \mathrm{~S}$ rRNA gene did not vary significantly between samples (data not shown) and was therefore proven to be a valid choice as a reference gene. The high similarity between both GH25 genes made it difficult to use primerbased methods to selectively analyze their expression. Therefore, flanking genes, including the genes encoding for the Fn3 proteins, were selected for RT-qPCR analysis and confirmed the upregulation of the larger cluster as observed in the microarray (Table 2). The RT-qPCR also confirmed the upregulation of the Fn3 gene (Blon_1825) in the smaller cluster.

\section{CONCLUSIONS}

The introduction of bovine GMP lead to a significant increase in the growth of the infant-associated strain, $B$. longum ssp. infantis. This effect was substantially reduced following periodate oxidation of the conjugated oligosaccharides. The inclusion of GMP as a functional ingredient may influence the predominance of beneficial bacteria in the gut. The effect was observed when $B$. longum ssp. infantis was cultured in a nutritionally complex environment; however, it will be key to determine if this growth promotion effect is evident in a mixed bacterial population as would occur in vivo. Treatment with GMP induced some glycogenes whereas it appeared to repress others, and these data suggest that the response of $B$. longum ssp. infantis to glycosylated substrates is a tightly regulated response and exposure to a specific glycan presentation elicits a highly specialized response. The transcriptional response may be divided into 3 quite distinct components: transport of extracellular molecules, glycan metabolism, and growth and cell division. It is likely that the transport and metabolism genes are specific to the GMP oligosaccharides whereas the growth and cell division response are likely to be general responses to increased growth rate.

\section{ACKNOWLEDGMENTS}

Noelle O'Riordan is in receipt of a Teagasc Walsh Fellowship. The authors graciously acknowledge the contribution of Paul W. O'Toole (School of Microbiology and Alimentary Pharmabiotic Centre, University College Cork, Ireland).

\section{REFERENCES}

Alemka, A., S. Whelan, R. Gough, M. Clyne, M. E. Gallagher, S. D. Carrington, and B. Bourke. 2010. Purified chicken intestinal mucin attenuates Campylobacter jejuni pathogenicity in vitro. J. Med. Microbiol. 59:898-903.

Altschul, S. F., W. Gish, W. Miller, E. W. Myers, and D. J. Lipman. 1990. Basic local alignment search tool. J. Mol. Biol. 215:403-410.

Bottacini, F., D. Medini, A. Pavesi, F. Turroni, E. Foroni, D. Riley, V. Giubellini, H. Tettelin, D. van Sinderen, and M. Ventura. 2010. Comparative genomics of the genus Bifidobacterium. Microbiology 156:3243-3254.

Chen, Q., J. Cao, Y. Jia, X. Liu, Y. Yan, and G. Pang. 2012. Modulation of mice fecal microbiota by administration of casein glycomacropeptide. Microbiol. Res. (Pavia) 3:e3.

da Silva, A. F., B. García-Fraga, J. López-Seijas, and C. Sieiro. 2014. Characterization and optimization of heterologous expression in Escherichia coli of the chitinase encoded by the chiA gene of Bacillus halodurans C-125. Process Biochem. 49:1622-1629. 
Delfour, A., J. Jolles, C. Alais, and P. Jolles. 1965. Caseino-glycopeptides: Characterization of a methionin residue and of the Nterminal sequence. Biochem. Biophys. Res. Commun. 19:452-455.

Edgar, R., M. Domrachev, and A. E. Lash. 2002. Gene Expression Omnibus: NCBI gene expression and hybridization array data repository. Nucleic Acids Res. 30:207-210.

Fournet, B., A. M. Fiat, J. Montreuil, and P. Jolles. 1975. The sugar part of kappa-caseins from cow milk and colostrum and its microheterogeneity. Biochimie 57:161-165.

Furlanetti, A. M., and L. F. Prata. 2003. Free and total GMP (glycomacropeptide) contents of milk during bovine lactation. Food Sci. Technol. (Campinas) 23:121-125.

Garrido, D., D. Barile, and D. A. Mills. 2012a. A molecular basis for bifidobacterial enrichment in the infant gastrointestinal tract. Adv. Nutr. 3:415S-421S.

Garrido, D., D. C. Dallas, and D. A. Mills. 2013a. Consumption of human milk glycoconjugates by infant-associated bifidobacteria: mechanisms and implications. Microbiology 159:649-664.

Garrido, D., J. H. Kim, J. B. German, H. E. Raybould, and D. A. Mills. 2011. Oligosaccharide binding proteins from Bifidobacterium longum ssp. infantis reveal a preference for host glycans. PLoS One 6:e17315.

Garrido, D., S. Ruiz-Moyano, R. Jimenez-Espinoza, H.-J. Eom, D. E. Block, and D. A. Mills. 2013b. Utilization of galactooligosaccharides by Bifidobacterium longum ssp. infantis isolates. Food Microbiol. 33:262-270.

Garrido, D., S. Ruiz-Moyano, D. G. Lemay, D. A. Sela, J. B. German, and D. A. Mills. 2015. Comparative transcriptomics reveals key differences in the response to milk oligosaccharides of infant gutassociated bifidobacteria. Sci. Rep. 5:13517.

Garrido, D., S. Ruiz-Moyano, and D. A. Mills. 2012b. Release and utilization of N-acetyl-D-glucosamine from human milk oligosaccharides by Bifidobacterium longum ssp. infantis. Anaerobe 18:430-435.

Gustavo Hermes, R., F. Molist, J. F. Pérez, A. G. de Segura, M. Ywazaki, R. Davin, M. Nofrarıas, T. K. Korhonen, R. Virkola, and S. M. Martın-Orúe. 2013. Casein glycomacropeptide in the diet may reduce Escherichia coli attachment to the intestinal mucosa and increase the intestinal lactobacilli of early weaned piglets after an enterotoxigenic E. coli K88 challenge. Br. J. Nutr. 109:1001-1012.

Hua, S., C. C. Nwosu, J. S. Strum, R. R. Seipert, H. J. An, A. M. Zivkovic, J. B. German, and C. B. Lebrilla. 2012. Site-specific protein glycosylation analysis with glycan isomer differentiation. Anal. Bioanal. Chem. 403:1291-1302.

Idota, T., H. Kawakami, and I. Nakajima. 1994. Growth-promoting effects of N-acetylneuraminic acid-containing substances on bifidobacteria. Biosci. Biotechnol. Biochem. 58:1720-1722.

Isoda, H., Y. Kawasaki, M. Tanimoto, S. Dosako, and T. Idota. 1989. Snow Brand Milk Products Co. Ltd., assignee. Use of compounds containing or binding sialic acid to neutralize bacterial toxins. European patent 385112 . EP Patent $0,385,118 \mathrm{~A} 2$.

Janer, C., C. Pelaez, and T. Requena. 2004. Caseinomacropeptide and whey protein concentrate enhance Bifidobacterium lactis growth in milk. Food Chem. 86:263-267.

Karav, S., A. Le Parc, J. M. Leite Nobrega de Moura Bell, S. A. Frese, N. Kirmiz, D. E. Block, D. Barile, and D. A. Mills. 2016. Oligosaccharides released from milk glycoproteins are selective growth substrates for infant-associated bifidobacteria. Appl. Environ. Microbiol. 82:3622-3630.

Kavanaugh, D. W., J. O'Callaghan, L. F. Buttó, H. Slattery, J. Lane, M. Clyne, M. Kane, L. Joshi, and R. M. Hickey. 2013. Exposure of Bifidobacterium longum ssp. infantis to milk oligosaccharides increases adhesion to epithelial cells and induces a substantial transcriptional response. PLoS One 8:e67224.

Kawasaki, Y., H. Isoda, M. Tanimoto, S. Dosako, T. Idota, and K. Ahiko. 1992. Inhibition by lactoferrin and kappa-casein glycomacropeptide of binding of cholera-toxin to its receptor. Biosci. Biotechnol. Biochem. 56:195-198.

Kawasaki, Y., H. Shinmoto, M. Tanimoto, S. Dosako, and T. Idota. 1993. Inhibition by k-casein glycomacropeptide and lactoferrin of influenza virus hemmaglutination. Biosci. Biotechnol. Biochem. $57: 1214-1215$.

Kilcoyne, M., J. Q. Gerlach, M. P. Farrell, V. P. Bhavanandan, and L. Joshi. 2011. Periodic acid-Schiff's reagent assay for carbohydrates in a microtiter plate format. Anal. Biochem. 416:18-26.

Kim, J. H., H. J. An, D. Garrido, J. B. German, C. B. Lebrilla, and D. A. Mills. 2013. Proteomic analysis of Bifidobacterium longum ssp. infantis reveals the metabolic insight on consumption of prebiotics and host glycans. PLoS One 8:e57535.

Leahy, S. C., D. Higgins, G. Fitzgerald, and D. v. Sinderen. 2005. Getting better with bifidobacteria. J. Appl. Microbiol. 98:1303-1315.

Little, E., P. Bork, and R. Doolittle. 1994. Tracing the spread of fibronectin type III domains in bacterial glycohydrolases. J. Mol. Evol. 39:631-643.

Livak, K. J., and T. D. Schmittgen. 2001. Analysis of relative gene expression data using real-time quantitative PCR and the 2(-Delta Delta C(T)). Methods 25:402-408.

LoCascio, R. G., P. Desai, D. A. Sela, B. Weimer, and D. A. Mills 2010. Broad conservation of milk utilization genes in Bifidobacterium longum ssp. infantis as revealed by comparative genomic hybridization. Appl. Environ. Microbiol. 76:7373-7381.

LoCascio, R. G., M. R. Ninonuevo, S. L. Freeman, D. A. Sela, R Grimm, C. B. Lebrilla, D. A. Mills, and J. B. German. 2007. Glycoprofiling of bifidobacterial consumption of human milk oligosaccharides demonstrates strain specific, preferential consumption of small chain glycans secreted in early human lactation. J. Agric. Food Chem. 55:8914-8919.

Nakajima, K., N. Tamura, K. Kobayashi-Hattori, T. Yoshida, Y. HaraKudo, M. Ikedo, Y. Sugita-Konishi, and M. Hattori. 2005. Prevention of intestinal infection by glycomacropeptide. Biosci. Biotechnol. Biochem. 69:2294-2301.

Neelima, R. Sharma, Y. S. Rajput, and B. Mann. 2013. Chemical and functional properties of glycomacropeptide (GMP) and its role in the detection of cheese whey adulteration in milk: a review. Dairy Sci. Technol. 93:21-43.

Ney, D. M., and M. R. Etzel. 2017. Designing medical foods for inherited metabolic disorders: Why intact protein is superior to amino acids. Curr. Opin. Biotechnol. 44:39-45.

Ney, D. M., B. M. Stroup, M. K. Clayton, S. G. Murali, G. M. Rice, F. Rohr, and H. L. Levy. 2016. Glycomacropeptide for nutritional management of phenylketonuria: A randomized, controlled, crossover trial. Am. J. Clin. Nutr. 104:334-345.

Ntemiri, A., F. N. Chonchuir, T. F. O'Callaghan, C. Stanton, R. P. Ross, and P. W. O'Toole. 2017. Glycomacropeptide sustains microbiota diversity and promotes specific taxa in an artificial colon model of elderly gut microbiota. J. Agric. Food Chem. 65:18361846.

O'Riordan, N., M. Kane, L. Joshi, and R. M. Hickey. 2014. Structural and functional characteristics of bovine milk protein glycosylation. Glycobiology 24:220-236.

Perrin, S., M. Warchol, J. P. Grill, and F. Schneider. 2001. Fermentations of fructo-oligosaccharides and their components by Bifidobacterium infantis ATCC 15697 on batch culture in semi-synthetic medium. J. Appl. Microbiol. 90:859-865.

Picard, C., J. Fioramonti, A. Francois, T. Robinson, F. Neant, and C. Matuchansky. 2005. Review article: Bifidobacteria as probiotic agents-physiological effects and clinical benefits. Aliment. Pharmacol. Ther. 22:495-512.

Robitaille, G. 2013. Growth-promoting effects of caseinomacropeptide from cow and goat milk on probiotics. J. Dairy Res. 80:58-63.

Saito, T., T. Itoh, and S. Adachi. 1981a. The chemical structure of a tetrasaccharide containing $\mathrm{N}$-acetylglucosamine obtained from bovine colostrum kappa-casein. Biochim. Biophys. Acta 673:487-494.

Saito, T., T. Itoh, S. Adachi, T. Suzuki, and T. Usui. 1981b. The chemical structure of neutral and acidic sugar chains obtained from bovine colostrum kappa-casein. Biochim. Biophys. Acta $678: 257-267$.

Saito, T., T. Itoh, S. Adachi, T. Suzuki, and T. Usui. 1982. A new hexasaccharide chain isolated from bovine colostrum kappa-casein 
taken at the time of parturition. Biochim. Biophys. Acta 719:309317.

Saito, T. I. T. 1980. The chemical structure of the main sugar moiety isolated from bovine whole casein. Agric. Biol. Chem. 44:10231030.

Sawin, E. A., T. J. De Wolfe, B. Aktas, B. M. Stroup, S. G. Murali, J. L. Steele, and D. M. Ney. 2015. Glycomacropeptide is a prebiotic that reduces Desulfovibrio bacteria, increases cecal short-chain fatty acids, and is anti-inflammatory in mice. Am. J. Physiol. Gastrointest. Liver Physiol. 309:G590-G601.

Scholtens, P. A. M. J., R. Oozeer, R. Martin, K. B. Amor, and J. Knol. 2012. The early settlers: Intestinal microbiology in early life. Annu. Rev. Food Sci. Technol. 3:425-447.

Sela, D. A., J. Chapman, A. Adeuya, J. H. Kim, F. Chen, T. R. Whitehead, A. Lapidus, D. S. Rokhsar, C. B. Lebrilla, J. B. German, N. P. Price, P. M. Richardson, and D. A. Mills. 2008. The genome sequence of Bifidobacterium longum ssp. infantis reveals adaptations for milk utilization within the infant microbiome. Proc. Natl. Acad. Sci. USA 105:18964-18969.

Sela, D. A., D. Garrido, L. Lerno, S. Wu, K. Tan, H. J. Eom, A. Joachimiak, C. B. Lebrilla, and D. A. Mills. 2012. Bifidobacterium longum ssp. infantis ATCC 15697 alpha-fucosidases are active on fucosylated human milk oligosaccharides. Appl. Environ. Microbiol. 78:795-803.

Sela, D. A., Y. H. Li, L. Lerno, S. A. Wu, A. M. Marcobal, J. B. German, X. Chen, C. B. Lebrilla, and D. A. Mills. 2011. An infantassociated bacterial commensal utilizes breast milk sialyloligosaccharides. J. Biol. Chem. 286:23620.

Tullio, L. T., E. N. L. Karkle, and L. M. B. Cândido. 2007. Review: Isolation and purification of milk whey glycomacropeptide. Boletim do Centro de Pesquisa de Processamento de Alimentos 25:121-132. 